\title{
Cell and neuron densities in the primary motor cortex of primates
}

\section{Nicole A. Young, Christine E. Collins and Jon H. Kaas*}

Department of Psychology, Vanderbilt University, Nashville, TN, USA

\section{Edited by:}

Gordon M. G. Shepherd,

Northwestern University, USA

Reviewed by:

Chet C. Sherwood, George

Washington University, USA

Guy Elston, Centre for Cognitive

Neuroscience, Australia

Kathleen S. Rockland

Massachusetts Institute of

Technology, USA

*Correspondence:

Jon H. Kaas, Department of

Psychology, Vanderbilt University,

301 Wilson Hall, 111 21st Avenue

South, Nashville, TN 37240, USA.

e-mail: jon.h.kaas@vanderbilt.edu
Cell and neuron densities vary across the cortical sheet in a predictable manner across different primate species (Collins et al., 2010b). Primary motor cortex, M1, is characterized by lower neuron densities relative to other cortical areas. M1 contains a motor representation map of contralateral body parts from tail to tongue in a mediolateral sequence. Different functional movement representations within M1 likely require specialized microcircuitry for control of different body parts, and these differences in circuitry may be reflected by variation in cell and neuron densities. Here we determined cell and neuron densities for multiple sub-regions of $\mathrm{M} 1$ in six primate species, using the semi-automated flow fractionator method. The results verify previous reports of lower overall neuron densities in M1 compared to other parts of cortex in the six primate species examined. The most lateral regions of $\mathrm{M} 1$ that correspond to face and hand movement representations, are more neuron dense relative to medial locations in $M 1$, which suggests differences in cortical circuitry within movement zones.

Keywords: M1, flow fractionator, isotropic fractionator, movement

\section{INTRODUCTION}

The cerebral cortex is a heterogeneous structure that contains multiple sensory and motor information-processing systems (e.g., Van Essen et al., 2011). A hallmark of the mammalian cerebral cortex is the regular arrangement of sensory and motor areas across its surface. Primary sensory areas are topographically organized to represent sensory receptor arrays and primary motor cortex has a general somatotopic organization of motor movement representations. It is reasonable to presume that numbers of cells and neurons in functionally distinct cortical areas vary according to information-processing demands. However, the majority of studies on this issue have only reported the total number of cells and neurons for the cerebral cortex as a whole (e.g. Pakkenberg and Gundersen, 1997; Christensen et al., 2007; Herculano-Houzel et al., 2008).

To date, only one study has detailed the total numbers of cells and neurons across the entire cortical expanse after dissection of the cortex into small tissue pieces (Collins et al., 2010b). This study not only demonstrated a clear, non-uniform distribution of cells and neurons across the cortex of all four primate species examined, it also illustrated a pattern of distribution of cells and neurons that was consistent across all of the primate species that were studied. The features of the typical primate pattern of cell and neuron distribution and number are (1) the highest cell and neuron densities are found in primary visual cortex, V1; (2) extrastriate cortical areas have relatively high cell and neuron densities; (3) primary auditory and somatosensory areas have relatively high cell and neuron densities compared to surrounding areas; and (4) motor cortex, M1, appears to have low neuron densities compared to other areas of cortex. These results are consistent with the earlier findings of Beaulieu and Colonnier (1989) who determined neuron number in the cortex below $1 \mathrm{~mm}^{2}$ of cortical surface in four visual areas, somatosensory area $3 \mathrm{~b}$, and two motor areas (4 gamma, 6a alpha) of the cortex of cats, and found that motor areas have the smallest number of neurons per column, while sensory areas contain more neurons, with the greatest number found in the binocular region of visual area 17. Skoglund et al. (1996) later investigated the number of neurons in primary motor, primary somatosensory and the second visual area in rats, and found significant differences in the number of neurons under a fixed amount of cortical surface area. They found that neuron density was highest in the second visual area (not V1), followed by primary somatosensory cortex. Primary motor cortex was the least neuron-dense cortical area. Despite the comprehensive evaluation of cortical cell and neuron number and distribution in the study by Collins and colleagues, the number of species examined in detail was limited and the dissection techniques used in that study had not been refined to allow evaluation of variation in cell and neuron density in different parts of topographically organized cortical areas, and in cases where functional areas were dissected from the cortex, areal boundaries were estimated from surface landmarks and sulcal patterns.

The present study was designed to examine the neuron densities in additional species of primates, to determine whether different representational zones within $\mathrm{M} 1$ have variable cell and neuron densities, and to compare estimated areal border locations with those determined electrophysiologically. We use the semiautomated flow fractionator method (Collins et al., 2010a; Young et al., 2012) to contrast M1 cell and neuron densities with the overall average densities across the cortex, and also to another specific primary cortical area, V1, to determine if M1 is contains relatively fewer neurons, as previously reported using other counting techniques. Here we also report estimates of neuron and total cell densities for different movement representation zones in 
M1 for three primate species. Variation in neuron and total cell densities within a sub-region of M1 may correspond to the type of movement produced in the sub-region.

\section{MATERIALS AND METHODS TISSUE}

Prosimian galago (Otolemur garnetti, $n=3$ ), New World owl monkey (Aotus nancymae, $n=1$ ), and squirrel monkey (Saimiri sciuresis, $n=1$ ) brains were obtained from ongoing experiments of other investigators at Vanderbilt University. Galago and New World monkey brains were perfused with $0.1 \mathrm{MPBS}$. Old World macaque (Macaca nemenstrina, $n=2$ ) and baboon (Papio cynocephalus anubis, $n=1$ ) brains were purchased from the Washington National Primate Research Center. An additional baboon (Papio hamadryas anubis, $n=1$ ) brain and a Hominid chimpanzee (Pan troglodytes, $n=1$ ) brain were purchased from the Texas Biomedical Research Institute. These brains were perfused with 0.1 MPBS, and shipped to us overnight in the same solution. All brains were bisected and one cortical hemisphere was separated from the subcortical structures, the pia was removed and the sulci were opened to flatten the cortical sheet. The flat hemispheres were fixed within $4 \%$ paraformaldehyde (PFA). Macaque 2, however, remained intact and was post-fixed in $4 \%$ PFA. M1 was dissected from the flattened hemispheres and separated from the remaining cortex after viewing the flattened hemispheres on a light box to identify myelin-dense sensory areas that appear darker relative to surrounding cortical areas, which is an effective means to quickly visualize cortical areas for this method of dissection (see Collins et al., 2010b; Campi et al., 2011). Boundaries of M1 were identified relative to the estimated boundaries of these areas, sulcal landmarks, and in reference to previously published studies that identify boundaries of M1 and its internal organization (Gould et al., 1986; Huang et al., 1988; Waters et al., 1990; Huntley and Jones, 1991; Donoghue et al., 1992; Gaspar et al., 1992; Nudo et al., 1992; Stepniewska et al., 1993, 2005; Preuss et al., 1997; Jain et al., 2000; Qi et al., 2000, 2010; Wu et al., 2000; Fang et al., 2008; Wong and Kaas, 2010; Gharbawie et al., 2011; Kaas, 2012). M1 boundaries in the intact brain of baboon case $09-04$ were identified according to intact sulcal landmarks and data from a previous report (Waters et al., 1990). In the galago, owl monkey, squirrel monkey, and baboon cases, the boundary of primary visual cortex (V1) is visible on the fixed brain surface, whether the cortex is flattened or not. Dissection cuts were placed along the visible, readily identifiable boundary. Remaining cortex in all species was dissected into approximately $5 \times 5 \mathrm{~mm}$ cortical pieces. All cortical pieces were weighed, and surface areas were measured when possible using freely available NIH Image J software (NIH, Bethesda, MD, USA).

\section{MOTOR AND SOMATOSENSORY MAPPING OF A GALAGO}

The primary motor map was derived for one galago (galago 3) using short-train intracortical microstimulation (ICMS). This mapping was undertaken to verify our estimation of M1 boundaries and its internal organization in comparison to estimates in other cases. Some sensory mapping was completed to further confirm the location of the caudal boundary of M1. Detailed methods for surgical preparation, and motor and sensory mapping, are described in previously published reports (see Wu et al., 2000; Wu and Kaas, 2003; Stepniewska et al., 2009; Qi et al., 2011). All surgical procedures were conducted in accordance with the National Institutes of Health Guide for the Care and Use of Laboratory Animals and with the approval and guidance of the Vanderbilt University Animal Care and Use Committee.

In brief, surgical mapping procedures were performed under isoflurane anesthesia. After the skull was opened, the dura was retracted and the cortex digitally photographed. Isoflurane anesthesia was replaced with ketamine hydrochloride diluted with physiological saline (1:4) delivered intravenously with an infusion pump to maintain a stable level of anesthesia $(30-50 \mathrm{mg} / \mathrm{kg} / \mathrm{h}$ ). Ketamine did not profoundly suppress cortical responsiveness. The blood vessel pattern on the cortical surface was used to guide electrode penetrations, which were marked on a printed copy of the digital photograph. The frontal cortex was explored with ICMS to identify the locations of the representations of body movements in area M1. In the ICMS procedure a stimulating electrode is lowered into the cortex to cortical layer 5 and the minimal electrical current necessary to elicit body movements is delivered to identify the motor representations for the cortical motor area. The microstimulation currents were delivered in 60-ms trains, with a pulse duration of $0.2 \mathrm{~ms}$, and a pulse frequency of $300 \mathrm{~Hz}$, which are the optimal parameters for eliciting movement in primates and other mammals (see Gould et al., 1986; Preuss et al., 1992; Wu et al., 2000; Young et al., 2011), without tissue damage. Stimulation was delivered with a low-impedance tungsten microelectrode inserted perpendicular to the cortical surface to a depth of $1.5-1.8 \mathrm{~mm}$. This depth was found to be optimal for eliciting responses in prosimian galagos (Wu et al., 2000). Stimulating currents were generated with a Master 8 stimulator (AMPI) with a biphasic stimulus isolator (Bak Electronics Inc.). The entire body of the animal was monitored for ICMS-evoked movements by two observers during the mapping session. The face representation area of M1 was defined by eliciting any movements involving the mouth, tongue, jaw, nose, ears, and eyelids. The forelimb representation area of M1 was defined by eliciting movements involving the shoulder, arm, elbow, wrist, and digits. The trunk representation included movements of the upper and middle torso. Movements were classified as hindlimb when movements of the tail, legs, foot, and toes were made. Somatosensory mapping was undertaken to better identify the caudal boundary of M1. Microelectrode penetrations were made in somatosensory cortex and the magnitudes of neuronal responses to tapping and manipulation of the body surface (face, forelimb, trunk, and hindlimb regions), as indicated by the acoustic strength of neuron firing audio output, were constantly evaluated as the microelectrode passed through the superficial to the middle layers of cortex (up to $1000 \mu \mathrm{m}$ depth). For each penetration, the receptive field location, size, and stimulus preference to light touch, tapping, and joint movement stimuli at the site where the strongest evoked response occurred were recorded on the photograph of the cortical surface. Boundaries of M1 and S1, and identifiable movement representations areas within $\mathrm{M} 1$, were marked with fluororuby tracer (FR) immediately before sacrifice to estimate their location on the cortical surface. Following euthanasia, the brain was removed and the right cortical hemisphere was manually 
flattened. The FR landmarks were used to conservatively dissect ICMS-derived M1 representations from remaining cortex for processing with the flow fractionator (Young et al., 2012).

\section{CELL AND NEURON DENSITY ESTIMATES}

The isotropic fractionator and flow fractionator cell and neuron counting methods were used to obtain cell and neuron estimates in cortical samples. Detailed processing steps for the isotropic fractionator and flow fractionator are described in previous reports (Herculano-Houzel and Lent, 2005; Collins et al., 2010a; Young et al., 2012). Cell and neuron estimates obtained by the isotropic fractionator and the flow fractionator are in excellent concordance (Collins et al., 2010a; Young et al., 2012), and therefore the two methods can be used interchangeably.

In brief, each cortical piece was homogenized using a glass Tenbroeck tissue grinder (Fisher Scientific) and a dissociation solution of sodium citrate and triton X-100 in distilled water. The resulting homogenized suspensions contained free-floating nuclei. The total suspension volumes were determined based on the sample density, resulting in suspension volumes between 2 and $6 \mathrm{ml}$. The total number of cells in a nuclear suspension was estimated using DNA staining with 4',6-diamidino-2phenylindole (DAPI) that fluoresces bright blue with ultraviolet excitation (460 nm emission). DAPI binds strongly to DNA and labels all nuclei in the suspension, regardless of cell type, thereby providing a means for determining cell number in the homogenized samples. The total cell number estimate includes all DAPIpositive cell types contained within the sample, including glia and endothelial cells, as well as neurons. Free-floating, DAPI-stained nuclei in samples from the main suspension were counted to estimate total cells using either fluorescence microscopy and a glass Neubauer counting chamber and matched coverslip (isotropic fractionator), or a fixed volume of $50 \mu \mathrm{l}$ of Countbright absolute counting beads (Invitrogen) was added to the sample prior to evaluation using a Becton Dickson (BD) 5-laser LSR II flow cytometer equipped with a $355 \mathrm{~nm}$ laser and using $\mathrm{BD}^{\mathrm{TM}}$ FACSDiva v. 6.1.3 software (flow fractionator). The total number of neurons in a nuclear suspension was estimated by immunolabeling a sample of the main sample suspension for neuronal nuclei with the anti-NeuN antibody (anti-neuronal nuclear antigen) (Millipore, Inc.) to determine the percentage of the total nuclei (DAPI+) that are also NeuN-immunoreactive (NeuN-IR). All samples went through epitope retrieval, which consisted of $30 \mathrm{~min}$ in $0.2 \mathrm{M}$ boric acid solution in an oven set at $70^{\circ} \mathrm{C}$. After epitope retrieval, samples were washed once with PBS then resuspended in PBS with primary antibody against NeuN added. Alexa Fluor 594 (AF594) goat anti-mouse IgG secondary antibody (Invitrogen, Inc.) was used to fluorescently tag NeuN-IR nuclei for counting on the fluorescence microscope (isotropic fractionator), and Alexa Fluor 647 (AF647) goat anti-mouse IgG secondary antibody (Invitrogen, Inc.) was used to estimate the proportion of NeuN-IR nuclei to the total population of DAPI+ nuclei on the flow cytometer. Detailed procedures for gating the flow cytometry data have been discussed elsewhere (see Collins et al., 2010a; Young et al., 2012). All flow cytometry experiments were conducted in the Vanderbilt University Medical Center Flow Cytometry Shared Resource.

\section{RESULTS}

Figure 1 illustrates the locations of M1 on the lateral aspect of the left cerebral hemisphere of the primate species examined in the present study, including prosimian galagos, New World owl monkeys and squirrel monkeys, Old World macaque monkeys and baboons and a Hominid chimpanzee. The location and organization of $\mathrm{M} 1$, and the rest of the cortical motor areas, are similar across these species despite differences in the size of the cortex.

\section{PROSIMIAN GALAGOS}

In the three galagos analyzed here, the total surface area of the flat cortex was $1782 \mathrm{~mm}^{2}$ (galago 1), $1850 \mathrm{~mm}^{2}$ (galago 2), and $1817 \mathrm{~mm}^{2}$ (galago 3). The total cortex weight for these individuals is also very consistent at 2.86, 2.87, and $2.37 \mathrm{~g}$ for galagos 1 , 2 , and 3, respectively. M1 was identified and dissected in galagos 1 and 2 by visual identification on a lightbox using myelination patterns as reference. Galago 3 (case 10-41) had motor map dissections borders determined by ICMS mapping of M1 (Figure 2). Our methods for M1 identification and dissection (lightbox vs. mapping) was shown to produce consistent M1 cell and neuron estimates across individuals, and therefore we believe both dissection methods can be used to accurately identify cortical areas. For example, while the total surface areas for galago $3\left(55 \mathrm{~mm}^{2}\right)$ and galago $2\left(78 \mathrm{~mm}^{2}\right)$ slightly varied, the mapped galago 3 had a total number of 12.7 million cells and 4.9 million neurons within the defined M1 area, while the lightbox-dissected galago 2 had a total number of 11.0 million cells and 4.5 million neurons within M1.

Table 1 shows the results averaged for all three galagos. In galagos, M1 comprised approximately $3.1 \%$ of the total cortical mass, and almost $3 \%$ of the total cortical surface area. The average cell density in M1 was 118 million cells/g or 181,000 cells per $\mathrm{mm}^{2}$ of cortical surface area. We found that the average proportion of DAPI-labeled nuclei that were also NeuN-IR was $32.2 \%$, meaning that $32.2 \%$ of nuclei in M1 were identified as neuronal. This resulted in the average neuron density in M1 of 29 million neurons/g or 68,742 neurons under $1 \mathrm{~mm}^{2}$ of cortical surface. To determine the magnitude of the cell and neuron density difference between M1 and V1 within each case, we calculated the average difference within a species for comparison with other species. The overall cortical cell density, averaged across all cortical areas and regions, including M1 and V1, in all three galagos, was 149 million cells/g or 205,398 cells per $\mathrm{mm}^{2}$ of cortical surface. In contrast with another primary cortical area, the average cell density in V1 was 241 million cells/g or 297,479 cells per $\mathrm{mm}^{2}$, the highest cell density in the cortex. The overall cortical neuron density across all areas was 64 million neurons/g or 86,231 neurons per $\mathrm{mm}^{2}$ of surface area. The average neuron density in V1 in galagos was 146 million neurons/g or 182,208 neurons per $\mathrm{mm}^{2}$ of surface area, approximately five times higher than the neuron density in M1 (29 million neurons/g and 68,742 neurons $/ \mathrm{mm}^{2}$ of cortical surface).

The M1 map derived by ICMS in galago 3 clearly illustrated a mediolateral pattern of the movement map from hindlimb to trunk, forelimb, and face and the boundaries between those movement representations (Figure 2A). Each representational zone in M1 was dissected in an effort to evaluate the mediolateral organization of the movement map. The dashed lines in 


\section{Otolemur garnettii}

(Galago)

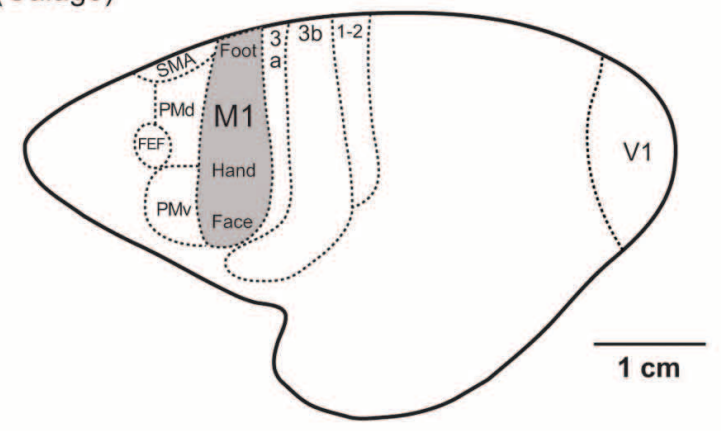

\section{Saimiri sciuresis}

(Squirrel Monkey)

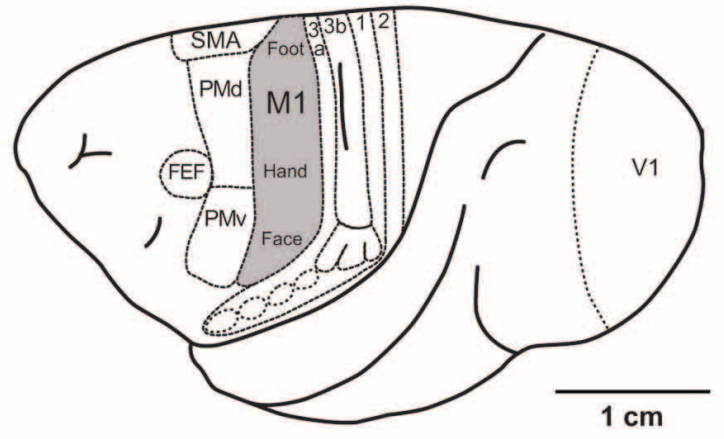

\section{Papio cynocephalus anubis}

(Baboon)

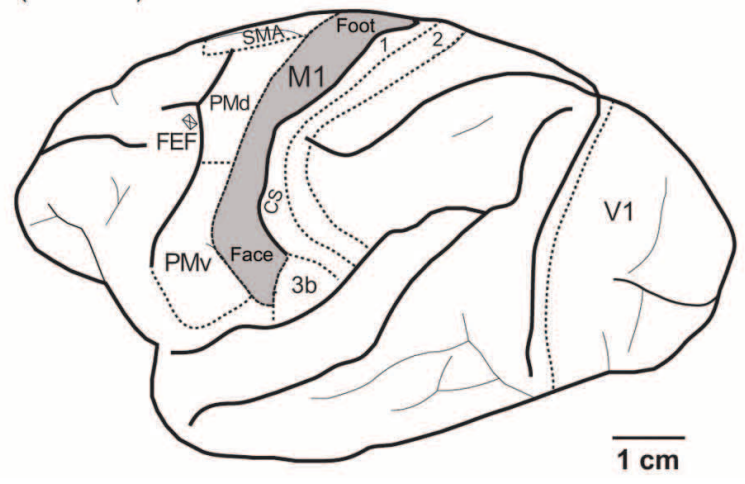

FIGURE 1 | The location of the primary motor cortex (M1) on the lateral view of the left cerebral hemisphere in several of the primate species discussed in this paper. The location and internal organization of $\mathrm{M} 1$ in New world and Old World monkeys (Gould et al., 1986; Huang et al., 1988; Waters et al., 1990; Huntley and Jones, 1991; Donoghue et al., 1992; Nudo et al.,

\section{Aotus nancymaae}

(Owl Monkey)

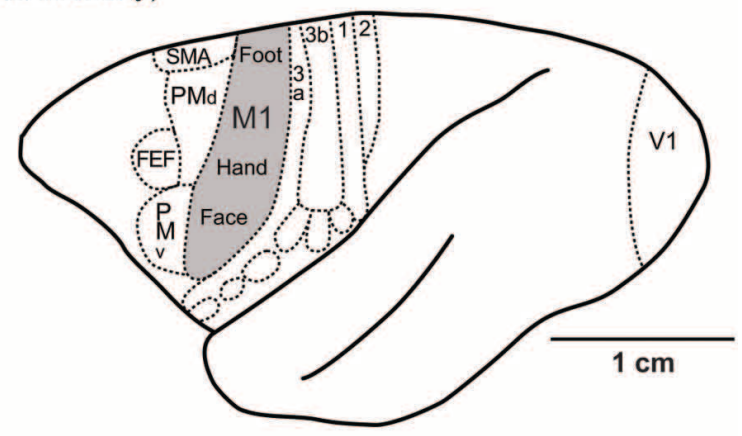

\section{Macaca nemenstrina}

(Macaque)

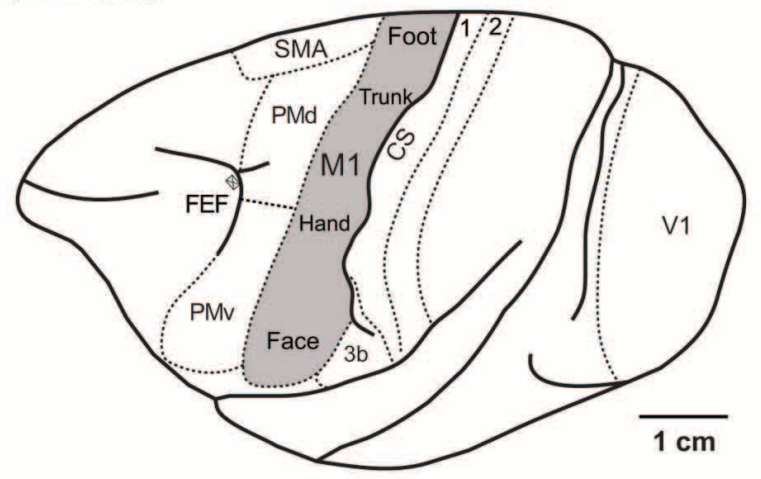

\section{Pan troglodytes}

(Chimpanzee)

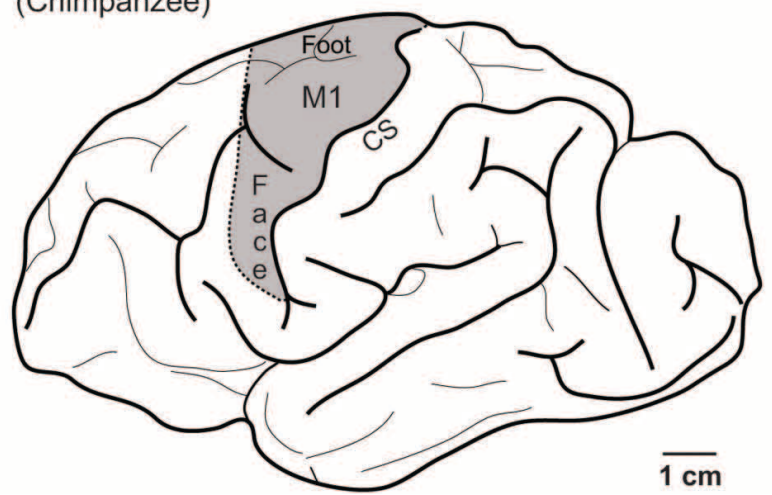

1992; Kaas, 2012) and prosimian galagos (Wu et al., 2000) consists of a fractured mosaic of smaller areas devoted to particular movements that are dispersed within a larger somatotopic framework of functional movement domains (see Kaas, 2012; Kaas et al., 2012 for review). Figure 1 shows the 


\section{FIGURE 1 | Continued}

locations of M1 in prosimian galagos (Wu et al., 2000; Wong and Kaas, 2010), New World owl monkeys (Gould et al., 1986) and squirrel monkeys

(Donoghue et al., 1992; Nudo et al., 1992), Old World macaques (Huntley and Jones, 1991; Preuss et al., 1997) and baboons (Waters et al., 1990), and

Great Ape chimpanzees (Bailey et al., 1950). Hindlimb movement

representations (foot) are located at the most dorsal-medial aspect of M1, and transitions to movements of the trunk of the body (trunk), forelimb and hand (hand), and finally face at the most ventral-lateral aspect of M1. Cortical areas located rostral to M1 include the dorsal (PMd) and ventral (PMv) premotor areas, the supplementary motor area (SMA), and frontal eye fields (FEF). Somatosensory areas of the anterior parietal cortex $(3 a, 3 b, 1,2)$ are caudal to M1. The location of the central sulcus (CS) is indicated in macaque, baboon, and chimpanzee.
Figures $\mathbf{2 B}, \mathbf{C}$ show where the dissection cuts were placed relative to the physiological motor map. Figure 2D illustrates the cell and neuron estimates for each sample according to its location and corresponding movement representation in M1. The data illustrated in the figure show that the more lateral regions of M1, which correspond to the face area, contain a higher density of neurons per $\mathrm{mm}^{2}$ of surface area than medial M1.

\section{OLD WORLD PRIMATES}

One macaque cortical hemisphere used in this study was manually flattened (macaque 1), while the cortical hemisphere of the other macaque brain (macaque 2) remained intact, thus surface area measurements were unavailable for macaque 2 . The total surface area of the flattened cortical hemisphere was $15,200 \mathrm{~mm}^{2}$ (macaque 1 ) and weighed $116.1 \mathrm{~g}$. M1 was visualized and dissected using the lightbox method for the flat cortical hemisphere in addition to the proximity to sulcal landmarks, while M1 in the intact cortical hemisphere (macaque 2) was estimated using sulcal patterns as landmarks based on previous reports (McGuinness et al., 1980; Wise and Tanji, 1981; Sessle and Wiesendanger, 1982; Huntley and Jones, 1991; Qi et al., 2000, 2010). Table 1 shows that the average M1 cell density in macaques is 75 million cells/g. The $\mathrm{M} 1$ cell density for macaque 1 alone was 84.1 million cells/g and the total cell density averaged across the entire cortex in case macaque 1 was 100 million cells/g. We found that the average proportion of DAPI-labeled nuclei that were also NeuN-IR was 30.9\% in macaque monkeys. The average neuron density in M1 was 23.5 million neurons/g for macaques. The M1 neuron density for macaque 1 alone was 22.3 million neurons/g. When averaged across all areas of cortex in macaque 1 , the overall neuron density was 48.0 million neurons/g.

For the baboons analyzed in this study, the total surface area of the flat cortex was $18,577 \mathrm{~mm}^{2}$ (baboon 1), and $23,400 \mathrm{~mm}^{2}$ (baboon 2). The total cortex weight for these individuals was fairly consistent at $50.0 \mathrm{~g}$ in baboon 1 and $56.4 \mathrm{~g}$ in baboon 2. M1 was identified and dissected in both baboon cases by visual identification on a lightbox using myelination patterns using sulcal landmarks as reference. Table 1 shows the summary of M1 data in both individuals. M1 comprised 3.55\% (baboon 2) and 4.20\% (baboon 1) of the total cortical mass, and $2.72 \%$ (baboon 2) and $3.52 \%$ (baboon 1 ) of the total cortical area. The average cell density in M1 was 80 million cells/g or 26,000 cells per $\mathrm{mm}^{2}$ of cortical surface for baboon 1 , and 72.6 million cells/g or 22,800 cells per $\mathrm{mm}^{2}$ for baboon 2 . The average proportion of DAPI-labeled nuclei that we found to also be NeuN-IR was $34.8 \%$ in baboon 1 and $38.6 \%$ in baboon 2, therefore the M1 neuron densities in these cases were 28.11 million neurons/g (90,000 neurons $\left./ \mathrm{mm}^{2}\right)$ and $28.10 \mathrm{million} / \mathrm{g}\left(88,000\right.$ neurons $\left./ \mathrm{mm}^{2}\right)$, respectively. These results were highly consistent across the two individuals.

We compared the M1 density averages to the overall cortical density average, and also compared them to the density averages for a readily identifiable primary sensory cortical area, V1 (primary visual cortex). The overall cortical cell density, averaged across all cortical areas and regions was 95.9 million cells/g or 251,603 cells per $\mathrm{mm}^{2}$ (baboon 1 ) and 78.3 million cells/g or 183,441 cells per $\mathrm{mm}^{2}$ (baboon 2 ). The neuron fraction across the cortex was $51 \%$ (baboon 1) and 54\% (baboon 2), therefore the overall neuron density across the cortex was 51.1 million neurons/g or 128,950 neurons per $\mathrm{mm}^{2}$ and 42.9 million neurons/g or 97,005 million neurons per $\mathrm{mm}^{2}$, respectively. To contrast the M1 cell and neuron density data with a primary sensory cortical area, the average cell density in V1 was 144 million cells/g or 283,307 cells per $\mathrm{mm}^{2}$ (baboon 1) and 139 million cells/g or 223,456 cells per $\mathrm{mm}^{2}$ (baboon 2). In V1, the fraction of DAPIlabeled nuclei that were also NeuN-IR was $75 \%$ (baboon 1 ) and $72 \%$ (baboon 2), therefore the V1 neuron densities in each case were 109 million neurons/g or 210,479 neurons per $\mathrm{mm}^{2}$ and 101 million neurons/g or 163,100 neurons per $\mathrm{mm}^{2}$, respectively. Cell and neuron densities in M1 were lower than the overall cortical average, and they were also much lower than cell and neuron density estimates in V1. In this data set, we report the baboon data separately as each case was a hybrid species of baboon, however, there was remarkable consistency in the overall, M1 and V1 data between the two cases in cell and neuron densities, showing a roughly 3-fold decrease in the cell density in M1 compared to $\mathrm{V} 1$, and an approximately 5 -fold difference in neuron density between M1 and V1.

In each baboon and macaque case, we estimated the locations of M1 movement representations and dissected areas according to those estimates. Without electrophysiological data to support our boundary estimates, we assigned dissected M1 cortical pieces to the likely lower limb, trunk, upper limb, hand, and face representations in a mediolateral sequence. The data in Figure 3 shows the distribution of cells and neurons within these representations for each individual, in a lateral-to-medial sequence from left to right. The neuron densities was found be higher in lateral parts of $\mathrm{M} 1$, corresponding to the face and hand representations, than in medial M1 (Figure 3D).

\section{OWL MONKEY, SOUIRREL MONKEY, AND CHIMPANZEE}

The total surface area of the flattened owl monkey cortex was $2000 \mathrm{~mm}^{2}$ with a total weight of $5.21 \mathrm{~g}$. The boundaries of M1 were estimated according to myelination patterns and cortical landmarks and was dissected and processed as a single sample. M1 comprised $5.12 \%$ of the total mass of the cortex and $8.6 \%$ 

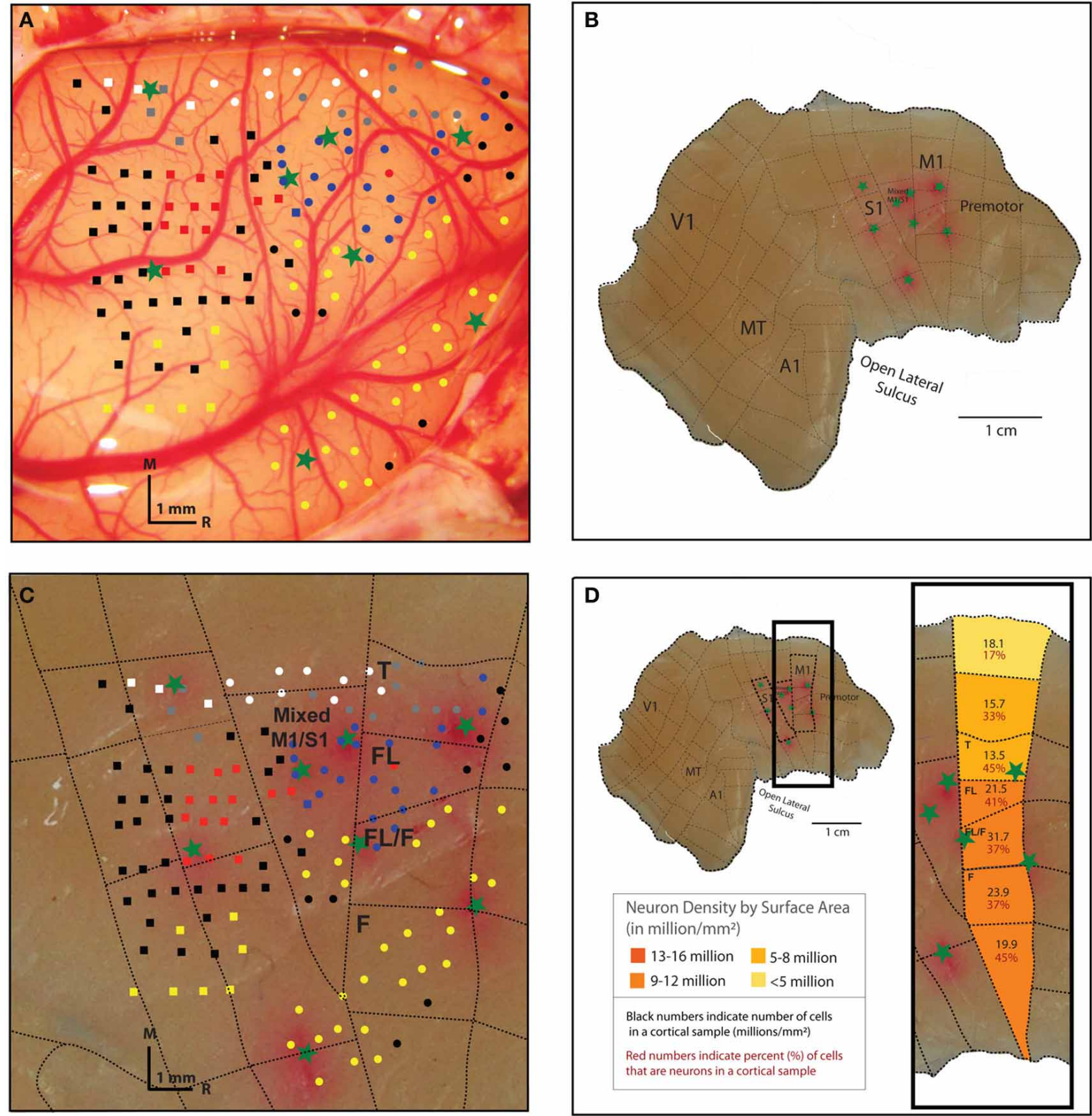

Neuron Density by Surface Area (in million $/ \mathrm{mm}^{2}$ )

13-16 million 5-8 million 9-12 million $\quad<5$ million

Black numbers indicate number of cells in a cortical sample (millions $/ \mathrm{mm}^{2}$ )

Red numbers indicate percent $(\%)$ of cells that are neurons in a cortical sample

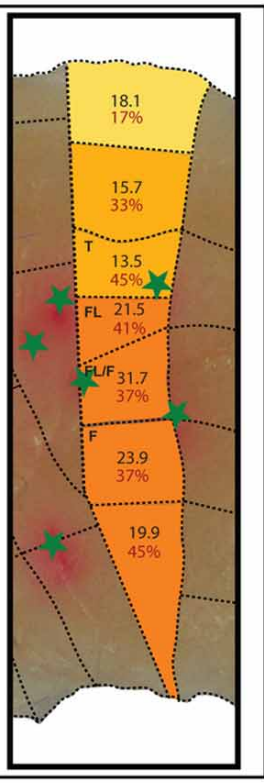

$\begin{array}{lll}\text { Injection } & \text { Trunk Receptive Field } & \text { Face Movement } \\ \text { Digit Receptive Field } & \text { No Sensory Response } & \text { Hindlimb Movement } \\ \text { Face Receptive Field } & \text { Forelimb Movement } & \text { Trunk Movement } \\ \square \text { Hindlimb Receptive Field } & \text { Digit Movement } & \text { No Motor Response }\end{array}$

\section{M: Medial}

R: Rostral

M1: Primary Motor Cortex

S1: Primary Sensory Cortex

$$
\begin{array}{ll}
\text { A1: Primary Auditory Cortex } & \text { FL: Forelimb } \\
\text { V1: Primary Visual Cortex } & \text { F: Face } \\
\text { MT: Medial Temporal Area } & \\
\text { T: Trunk } &
\end{array}
$$

FIGURE 2 | The right cortical hemisphere of a galago (galago 3) was exposed, and movements were evoked at cortical sites in M1 with 60-ms microstimulation trains delivered to layer $\mathbf{5}(\mathbf{A})$. The locations of stimulation sites where movements were elicited are indicated by color-coded dots on the cortical surface. Somatosensory mapping of receptive fields (layer 4) responsive to tactile stimulation are indicated by color-coded squares on the cortical surface. Somatosensory mapping was undertaken to better identify the caudal boundary of M1. Boundaries of M1 and S1, and identifiable movement representations areas within $\mathrm{M} 1$, were marked with a fluororuby tracer (FR) immediately before sacrifice to estimate their location on the cortical surface (green stars). Following sacrifice, the brain was removed and the right cortical hemisphere was manually flattened (B). The FR-indicated areas were used as guidelines to conservatively dissect microstimulation-derived M1representations from remaining cortex. Dashed lines indicate dissections lines that resulted in 84 pieces for the entire cortical hemisphere. The dissection resulted in four pieces identified as $\mathrm{M} 1$ by microstimulation mapping (C). Areas dedicated to movements of the trunk, forelimb, and face were readily identified and efforts were made to dissect along these movement representations boundaries within M1. Each M1 piece, as well as cortical pieces medial and lateral to M1, had cell and neuron densities estimated using the flow fractionator method (D). The most lateral aspect of $\mathrm{M} 1$, which includes facial movement representations, is the most neuron dense area. 


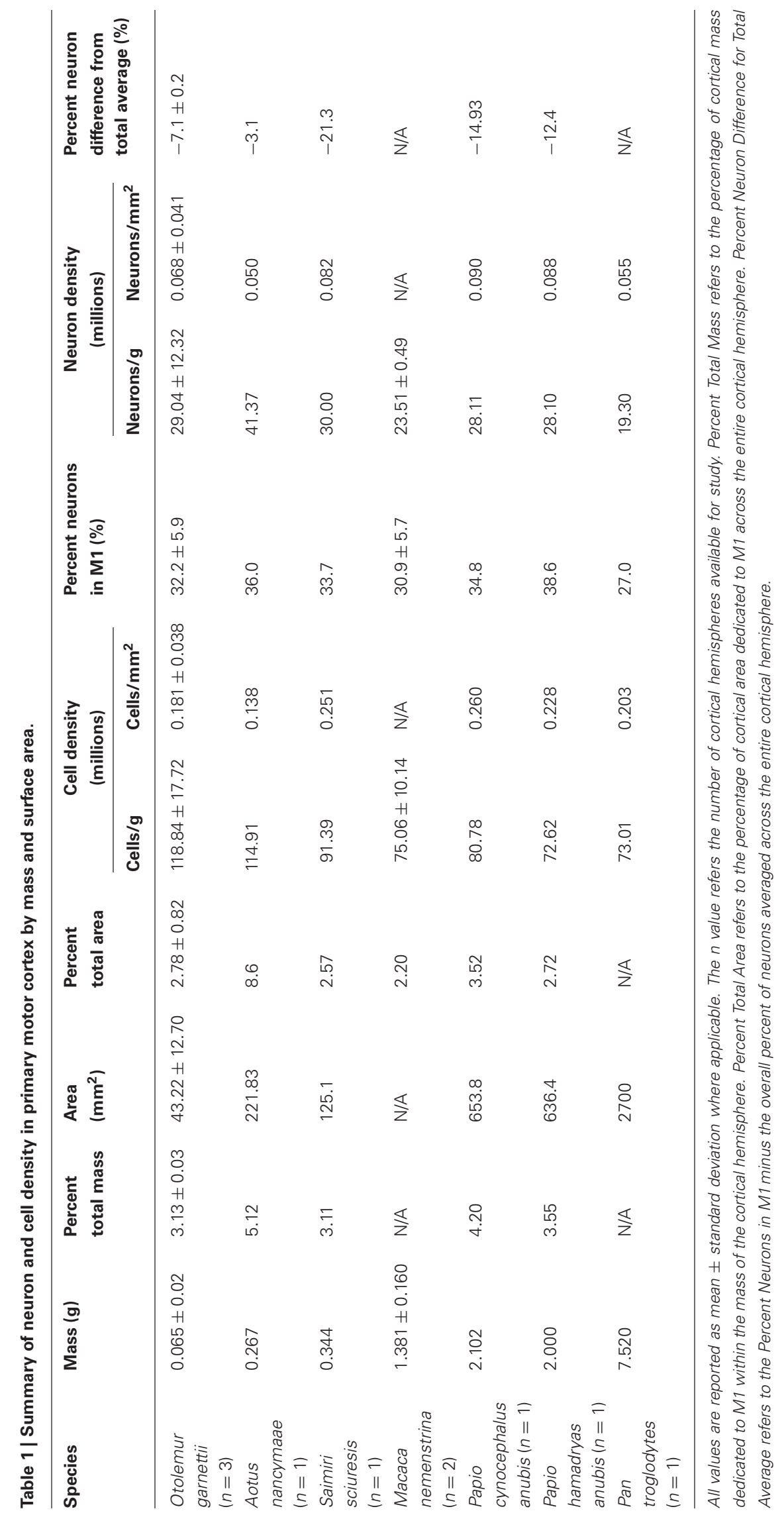




\section{A Cell Density by Mass}

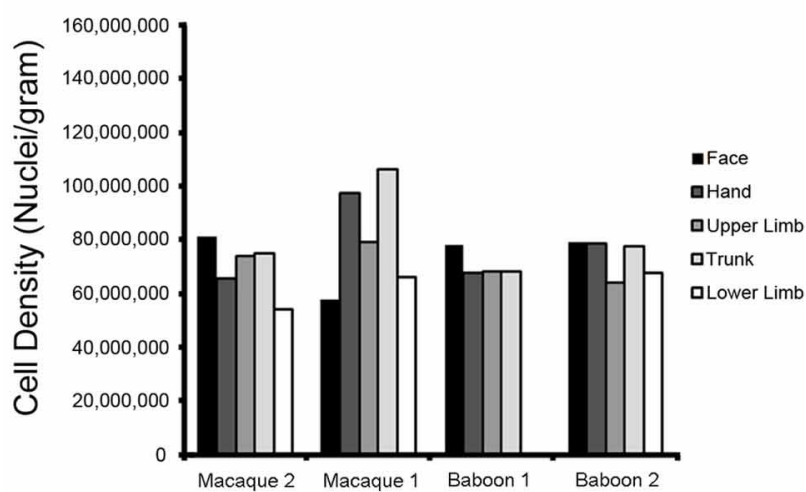

c Neuron Density by Mass

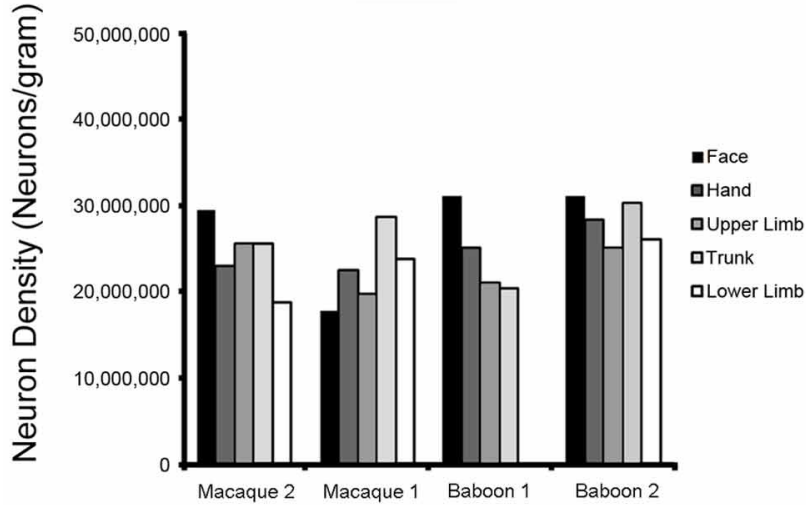

FIGURE 3 | Histograms of cell and neuron density in M1 of Old World monkeys. Movement representation boundaries within M1 were estimated, the motor cortex was dissected along those boundaries, and cell and neuron densities were obtained using the flow fractionator. The results show that distribution of cells by mass $(\mathbf{A})$ and by surface area

(B) does not vary substantially within $\mathrm{M} 1$ in either the macaque

\section{в Cell Density by Surface Area}

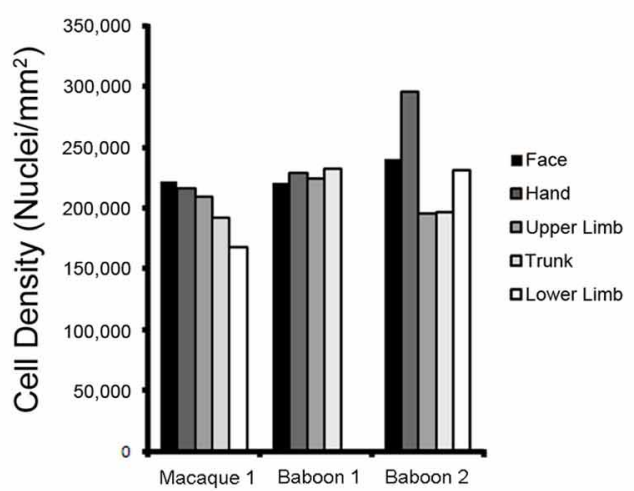

D Neuron Density by Surface Area

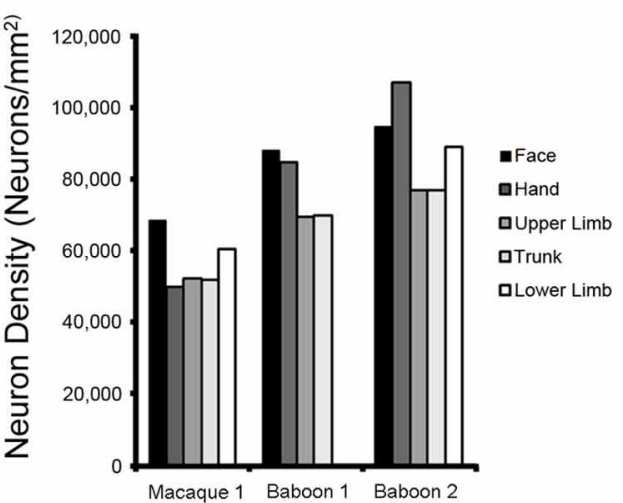

(macaque 1; macaque 2) or baboon (baboon 1; baboon 2). Neuron density by mass (C) and surface area (D) shows a trend toward the most lateral aspect within $\mathrm{M} 1$, which includes face and hand movement

representations, as being the most neuron dense area in both species. Please note that macaque 2 was an intact dissection and, as a result, cortical surface area could not be accurately estimated. of the total cortical surface area. The M1 cell and neuron density by weight are approximately 115 million cells/g and 41 million neurons/gram of cortical tissue. The $\mathrm{M} 1$ cell and neuron densities per $\mathrm{mm}^{2}$ of cortical surface are 138,000 cells $/ \mathrm{mm}^{2}$ and 50,000 neurons $/ \mathrm{mm}^{2}$. The overall cell density averaged across the entire cortex was 139 million cells/g or 276,595 cells per $\mathrm{mm}^{2}$. The overall proportion of neurons was $39 \%$, compared to the M1 neuron proportion of $36 \%$, resulting in an overall neuron density of 55.6 million neurons/g or 106,227 neurons per $\mathrm{mm}^{2}$. The V1 cell density in this case was 165 million cells/g or 270,703 cells per $\mathrm{mm}^{2}$. The proportion of cells in V1 that were NeuN-IR was 58\%, resulting in a neuron density of 96.7 million neurons/g or 158,554 neurons per $\mathrm{mm}^{2}$ of primary visual cortex. V1 has approximately a two-fold higher neuron density than M1.

The flattened squirrel monkey cortex had a total surface area of $4900 \mathrm{~mm}^{2}$ and weighted $11.1 \mathrm{~g}$. M1 comprised $3.11 \%$ of the total mass of the cortex and $2.57 \%$ of the total cortical surface area.
Cell and neuron densities by weight are 91 million cells/g and 30 million neurons/g. Cell and neuron densities by surface area are 251,000 cells $/ \mathrm{mm}^{2}$ and 82,000 neurons $/ \mathrm{mm}^{2}$ of cortical surface area (Table 1). The overall cell density averaged across the entire cortex was 134 million cells/g or 279,862 cells per $\mathrm{mm}^{2}$. With an overall neuron fraction of $55 \%$, the overall cortical neuron density was 79.5 million neurons/g or 159,457 neurons per $\mathrm{mm}^{2}$. V1 cell density was 202 million cells/g or 345,197 cells per $\mathrm{mm}^{2}$. With a V1 neuron fraction of $75 \%$, this resulted in a neuron density of 152 million neurons/g or 255,897 neurons per $\mathrm{mm}^{2}$ of primary visual cortex. M1 neuron density is approximately five times lower than the neuron density in V1, and over two times lower than average cortical density.

In the chimpanzee, M1 weighted $7.25 \mathrm{~g}$ and had a surface area of $2700 \mathrm{~mm}^{2}$. The cell and neuron densities for M1 are 73 million cells/g and 19 million neurons/g. The cell and neuron densities for M1 by surface area are 203,000 
cells $/ \mathrm{mm}^{2}$ and 55,000 neurons $/ \mathrm{mm}^{2}$ (Table 1). M1 contains about $27 \%$ neurons overall. V1 data are not yet available for this species.

\section{DISCUSSION}

Here we demonstrate that tissue boundaries for cortical areal dissections are readily determined by electrophysiological mapping, and that estimates of areal boundaries based on cortical landmarks also result in reasonably accurate dissections. The average percentage of NeuN-immunoreactive cells in M1 was fairly consistent across all six species of primates reported here, ranging from approximately $27 \%$ neurons in the chimpanzee to $36 \%$ neurons in the baboon. From the present dataset, it appears that primary motor cortex is approximately 2-5 times less neurondense than primary visual cortex and 1.4-2.7 times less dense than cortex overall in the species examined here. Four of the five species examined here showed a 5-fold difference in M1:V1 neuron density, with the exception being the nocturnal owl monkey that had a two-fold difference. The owl monkey, however, is the only species examined here to have an overall neuron density in $\mathrm{V} 1$ that is less than 100 million neurons/g of cortical tissue, making it unusual in comparison to the other primates. In species where it was possible to dissect M1 according to motor representations, there appears to be a trend toward a higher density of neurons in more lateral regions of M1, representing hand and face, compared to more medial areas representing trunk and hindlimb. These data, however, are based on one case where representational boundaries were defined electrophsyiologically and four other cases in which the boundaries were approximated using cortical landmarks.

\section{CELLS AND NEURON DENSITIES IN M1 IN PRIMATES}

The locations and organizations of M1 are similar across the six species of primates we have examined, and between individuals within a species, which suggests that the primate pattern of primary motor cortex emerged early in primate evolution, and has been conserved in the prosimian and simian branches within the primate order. We have shown that the proportion of mass and surface area dedicated to M1 remains relatively constant across species, despite the roughly 15 -fold variation in total cortical surface area across the species examined.

\section{ARCHITECTURE AND NEURON DENSITY}

In a previous study, we demonstrated that primate M1 has a distinctively lower neuron density relative to other areas and regions of cortex and that the primate cortex is not uniform in its distribution of neurons across areas and regions (Collins et al., 2010b). These results are consistent with those of other reports in which the lowest neuron densities in the cortex reported for cats and rats were also in the primary motor area (Beaulieu and Colonnier, 1989; Skoglund et al., 1996). Our data extend these findings to primates. In addition, our findings illustrating variations in neuron density by representational zone in M1 are analogous to the previous finding of the highest neuron density in V1 of cats, occurring in the binocular representational zone (Beaulieu and Colonnier, 1989). In contrast to these consistent findings for M1 across rats, cats and a range of primates, a recent report including several species reported similar neuron densities for all cortical areas, including M1, except for V1 of primates (Carlo and Stevens, 2013). This study basically repeated the earlier observations of Rockel et al. (1980). We do not know the reasons for such contradictory findings, except to note that both of the studies used extremely sparse sampling, and the methods for identifying cortical areas were unclear.

One reason for a real differences in neuron density appears to be layer 4 . The neuron dense layer of tightly packed, small neurons is remarkably thin and nearly indistinguishable in primary motor cortex, M1. Beaulieu and Colonnier (1989) concluded that areas in cats with the highest neuron densities tended to be sensory areas with a wide, neuron-dense layer IV, whereas motor areas had lower neuron densities and a significantly reduced layer 4. Variations in neuron densities across areas and regions of the cortex have been attributed to differences in developmental programs, with primary sensory areas having higher neuron densities (Dehay and Kennedy, 2007) and rostral portions of the cerebral cortex generally having a lower overall neuron density relative to more caudal cortical areas (Cahalane et al., 2012).

In addition to having a thin or missing layer of small granule cells, M1 is characterized by some of the largest of pyramidal cells (e.g., Stepniewska et al., 1993; Preuss et al., 1996, 1997). The sizes of pyramidal cells also differ across cortical areas (e.g., see Elston and Rosa, 1997; Elston and Rockland, 2002; Elston et al., 2005a,b; Bianchi et al., 2012) in ways that likely account for different functional capabilities. For example, spine densities on the basal dendritic arbors of pyramidal neurons in layer III of granular prefrontal cortex (gPFC) are as much as 16 times greater than spine densities on layer III pyramidal cells in V1 (Elston et al., 2006). These large neurons are more densely interconnected and potentially sum more sources of inputs within their more complex dendritic arbors (Elston, 2007). In general, small cells are activated by few inputs and thereby preserve information, while large pyramidal neurons sum many inputs and have integrated functions (Kaas, 2000).

The agranular and dysgranular cortex of M1 is comprised mostly of large pyramidal neurons in prominent layers 3 and 5, with some pyramidal cells in layer 5 being Betz cells, the largest neurons in the cortex, with diameters as large as 100 microns. Given the relatively lower density of neurons in primary motor cortex in the primates examined here, particularly in relation to the extremely high neuron densities in V1 where the neurons are extremely small, it seems reasonable to surmise that neuron density and neuron size may co-vary. However, previous research has examined the relationship between soma size and dendritic arbor size, concluding that the largest cells do not necessarily have the largest dendritic arbors (Elston and Rockland, 2002). Moreover, the spine density of neurons in M1 is significantly higher than that in V1 layer III pyramidal cells, but is lower than in pyramidal cells in layer III of premotor area 6 (Elston and Rockland, 2002; Elston, 2007).

\section{HETEROGENEITY WITHIN M1 IN PRIMATES}

We have previously demonstrated that M1 in primates is a region that, as a whole, is neuron sparse relative to the rest of cortex (Collins et al., 2010b). Here we demonstrate that it is also a heterogeneous structure that varies in neuron density across different 
representational zones, with lower neuron densities in medial M1 and higher neuron densities in lateral M1.

More medial M1 representing movements of the lower body is also more myelinated, possibly because corticospinal projections of the lower body movement representations in medial M1 are more heavily myelinated because they traverse longer distances to reach their more distant spinal cord targets (Glasser and Van Essen, 2011). Heavier myelination, in combination with a larger axon, would speed conductance to long-range targets. Hand and face body movement representations of lateral M1 would be less heavily myelinated due to closer proximity of their targets. In general, neurons with longer, thicker axons have larger cell bodies that reduce overall neuron densities.

Another possibility is that the higher neuron densities in lateral M1 reflect higher numbers of modulatory GABAergic interneurons. During the execution of voluntary movements, fast-spiking parvalbumin (PV)-expressing GABAergic interneurons are active in motor cortex, which suggests that they play a role in shaping ongoing movements (Isomura et al., 2009). The distribution and pattern of GABAergic immunoreacitve neurons in the visual areas processing areas (DeFelipe et al., 1999), and prefrontal cortex (Elston and Gonzalez-Albo, 2003) has been thought to vary with regional specializations related to information processing demands. The M1 face representation in hominids has a greater number of $\mathrm{PV}$-immunoreactive neurons relative to
Old World species, and this modification in face representation microcircuitry may be involved in supporting sophisticated coordination of facial muscles (Sherwood et al., 2004). A higher number of modulatory interneurons in face and hand representations may reflect an increased capacity for fine motor control, allowing the social advantage of better and increased skill in manipulating the external environment with the hands. The distribution of GABAergic subtypes have been characterized within specific movement representations and compared across species (Sherwood et al., 2003, 2004) or across other cortical areas outside M1 (Sherwood et al., 2010), but no study has yet examined the GABAergic neuron distribution within the mediolateral sequence of M1 movement representations.

\section{ACKNOWLEDGMENTS}

This work was supported by a grant from the G. Harold and Leila Y. Mathers Foundation to Jon H. Kaas and Christine E. Collins. Flow cytometry experiments were conducted in the Vanderbilt Medical Center (VMC) Flow Cytometry Shared Resource. The VMC Flow Cytometry Shared Resource is supported by the Vanderbilt Ingram Cancer Center (P30 CA68485) and the Vanderbilt Digestive Disease Research Center (DK058404). The authors would like to thank Dr. Iwona Stepniewska, Dr. Hui-Xin Qi, David Flaherty, Laura Trice, Feyi Aworunse, and Kallie Foust for assistance.

\section{REFERENCES}

Bailey, P., Von Bonin, G., and McCulloch, W. S. (1950). The Isocortex of the Chimpanzee. Urbana, IL: University of Illinois Press.

Beaulieu, C., and Colonnier, M. (1989). Number of neurons in individual laminae of areas 3B, 4 gamma, and $6 \mathrm{a}$ alpha of the cat cerebral cortex: a comparison with major visual areas. J. Comp. Neurol. 279, 228-234.

Bianchi, S., Stimpson, C. D., Bauernfeind, A. L., Schapiro, S. J., Baze, W. B., McArthur, M. J., et al. (2012). Dendritic morphology of pyramidal neurons in the chimpanzee neocortex: regional specializations and comparison to humans. Cereb. Cortex. doi: 10.1093/cercor/bhs239. [Epub ahead of print].

Cahalane, D. J., Charvet, C. J., and Finlay, B. L. (2012). Systematic, balancing gradients in neuron density and number across the primate isocortex. Front. Neuroanat. 6:28. doi: 10.3389/fnana.2012.00028

Campi, K. L., Collins, C. E., Todd, W. D., Kaas, J., and Krubitzer, L. (2011). Comparison of area 17 cellular composition in laboratory and wild-caught rats including diurnal and nocturnal species. Brain Behav. Evol. 77, 116-130.
Carlo, C. N., and Stevens, C. F. (2013). Structural uniformity of the neocortex, revisited. Proc. Natl. Acad. Sci. U.S.A. 110, 1488-1493.

Christensen, J. R., Larsen, K. B., Lisanby, S. H., Scalia, J., Arango, V., Dwork, A. J., et al. (2007). Neocortical and hippocampal neuron and glial cell numbers in the rhesus monkey. Anat. Rec. 290, 330-340.

Collins, C. E., Young, N. A., Flaherty, D. K., Airey, D. C., and Kaas, J. H. (2010a). A rapid and reliable method for counting neurons and other cells in brain tissue: a comparison of flow cytometry and manual counting methods. Front. Neuroanat. 4:5. doi: 10.3389/neuro.05.005.2010

Collins, C. E., Airey, D. C., Young, N. A., Leitch, D. B., and Kaas, J. H. (2010b). Neuron densities vary across and within cortical areas in primates. Proc. Natl. Acad. Sci. U.S.A. 107, 15927-15932.

DeFelipe, J., González-Albo, M. C., del Río, M. R., and Elston, G. N. (1999). Distribution and patterns of connectivity of interneurons containing calbindin, calretinin and parvalbumin in visual areas of the occipital and temporal lobes of the macaque monkey. J. Comp. Neurol. $412,515-526$
Dehay, C., and Kennedy, H. (2007). Cell-cycle control and cortical development. Nat. Rev. Neurosci. 8, 438-450.

Donoghue, J. P., Liebociv, S. J., and Sanes, J. N. (1992). Organization of the forelimb area in squirrel monkey motor cortex: representation of individual digit, wrist, and elbow muscles. Exp. Brain Res. 89, 1-19.

Elston, G. N. (2007). "Specialization of the neocortical pyramidal cell during primate evolution," in Evolution of the Nervous System: A Comprehensive System Reference, ed J. H. Kaas (Oxford, UK: Elsevier Inc), 191-242.

Elston, G. N., Benavides-Piccione, R., Elston, A., DeFelipe, J., and Manger, P. (2005a). Specialization in pyramidal cell structure in the sensory-motor cortex of the vervet monkey (Cercopithecus pygerythrus). Neuroscience 134 , 1057-1068.

Elston, G. N., Benavides-Piccione, R., Elston, A., Manger, P., and DeFelipe, J. (2005b). Specialization in pyramidal cell structure in the sensorymotor cortex of the Chacma baboon (papio ursinus) with comparative notes on the macaque monkey. Anat. Rec. A Discov. Mol. Cell. Evol. Biol. 286, 854-865.

Elston, G. N., Benavides-Piccione, R., Elston, A., Zietsch, B., Defelipe,
J., Manger, P., et al. (2006). Specializations of the granular prefrontal cortex of primates: implications for cognitive processing. Anat. Rec. A Discov. Mol. Cell. Evol. Biol. 288, 26-35.

Elston, G. N., and Gonzalez-Albo, M. C. (2003). Parvalbumin-, calbindin-, and calretininimmunoreactive neurons in the orefrontal cortex of the owl monkey (Aotus trivirgatus): a standardized quantitative comparsion with sensory and motor areas. Brain Behav. Evol. 62, 19-30.

Elston, G. N., and Rockland, K. (2002). The pyramidal cell of the sensorimotor cortex of the macaque monkey: phenotypic variation. Cereb. Cortex 12, 1071-1078.

Elston, G. N., and Rosa, M. G. P. (1997). The occipitoparietal pathway of the macaque monkey: comparison of pyramidal cell morphology in layer III of functionally related cortical visual areas. Cereb. Cortex 7 , 432-452.

Fang, P. C., Stepniewska, I., and Kaas, J. H. (2008). Corpus callosum connections of subdivisions of motor and premotor cortex, and frontal eye field in a prosimian primate, Otolemur garnetti. J. Comp. Neurol. 508, 565-578.

Gaspar, P., Stepniewska, I., and Kaas, J. H. (1992). Topography and 
collateralization of the dopaminergic projections to motor and lateral prefrontal cortex in owl monkeys. J. Comp. Neurol. 325, 1-21.

Gharbawie, O. A., Stepniewska, I., Qi, H. X., and Kaas, J. H. (2011). Multiple parietal-frontal pathways mediate grasping in macaque monkeys. J. Neurosci. 31, 11660-11677.

Glasser, M. F., and Van Essen, D. C. (2011). Mapping human cortical areas in vivo based on myelin content and revealed by $\mathrm{T} 1$ - and T2-weighted MRI. J. Neurosci. 31, 11597-11616.

Gould, H. J., Cusick, C. G., Pons, T. P., and Kaas, J. H. (1986). The relationship of the corpus callosum connections to electrical stimulation maps of the motor, supplementary motor, and the frontal eye fields in owl monkeys. J. Comp. Neurol. 247, 297-325.

Herculano-Houzel, S., Collins, C. E., Wong, P., Kaas, J. H., and Lent, R. (2008). The basic non-uniformity of the cerebral cortex. Proc. Natl. Acad. Sci. U.S.A. 105, 12593-12598.

Herculano-Houzel, S., and Lent, R. (2005). Isotropic fractionator: a simple, rapid method for the quantification of total cell and neuron numbers in the brain. J. Neurosci. 25, 2518-2521.

Huang, C. S., Sirisko, M. A., Hiraba, H., and Murray, G. M. (1988). Organization of the primate face motor cortex as served by intracortical microstimulation and electrophysiological identification of afferent inputs and corticobulbar projections. J. Neurophysiol. 59, 796-818.

Huntley, G. W., and Jones, E. G. (1991). Relationship of intrinsic connections to forelimb movement representations in monkey motor cortex: a correlative anatomical and physiological study. J. Neurophysiol. 66, 390-413.

Isomura, Y., Harukuni, R., Takekawa, T., Aizawa, H., and Fukai, T. (2009). Microcircuitry coordination of cortical motor information in selfinitiation of voluntary movements. Nat. Neurosci. 12, 1586-1593.

Jain, N., Qi, H. X., Catania, K. C., and Kaas, J. H. (2000). Anatomical correlates of the face and oral cavity representations in the somatosensory cortical area $3 \mathrm{~b}$ on monkeys. J. Comp. Neurol. 429, 455-468.

Kaas, J. H. (2000). Why is brain size so important: design problems and solutions as neocortex gets bigger or smaller. Brain Mind 1, 7-23.
Kaas, J. H. (2012). Evoultion of columns, modules, and domains in the neocortex of primates. Proc. Natl. Acad. Sci. U.S.A. 109, 10655-10660.

Kaas, J. H., Gharbawie, O. A., and Stepniewska, I. (2012). Cortical networks for ethologically relevant behaviors in primates. Am. J. Primatol. doi: 10.1002/ajp.22065. [Epub ahead of print].

McGuinness, E., Sivertsen, D., and Allman, J. M. (1980). Organization of the face representation in macaque motor cortex. J. Comp. Neurol. 193, 591-608.

Nudo, R. J., Jenkins, W. M., Merzenich, M. M., Prejean, T., and Grenda, R. (1992). Neurophysiological correlates of the hand preference in primary motor cortex of adult squirrel monkeys. J. Neurosci. 12, 2918-2947.

Pakkenberg, B., and Gundersen, H. J. (1997). Neocortical neuron number in humans: effect of sex and age. J. Comp. Neurol. 384, 312-320.

Preuss, T. M., Stepniewska, I., Jain, N., and Kaas, J. H. (1997). Multiple divisions of macaque precentral motor cortex identified with neurofilament antibody SMI32. Brain Res. 767, 148-153.

Preuss, T. M., Stepniewska, I., and Kaas, J. H. (1992). Microstimulation studies of motor cortical organization in lorisid primates. Eur. J. Neurosci. Suppl. 5:174.

Preuss, T. M., Stepniewska, I., and Kaas, J. H. (1996). Movement representation in the dorsal and ventral premotor areas of owl monkeys: a microstimulation study. J. Comp. Neurol. 371, 649-676.

Qi, H.-X., Gharbawie, O. A., Wong, P., and Kaas, J. H. (2011). Cell-poor septa separate representations of digits in the ventroposterior nucleus of the thalamus in monkeys and prosimian galagos. J. Comp. Neurol. 519, 738-758.

Qi, H. X., Jain, N., Collins, C. E., Lyon, D. C., and Kaas, J. H. (2010). Functional organization of motor cortex of adult macaque monkeys is altered by sensory loss in infancy. Proc. Natl. Acad. Sci. U.S.A. 107, 3192-3197.

Qi, H. X., Stepniewska, I., and Kaas, J. H. (2000). Reorganization of primary motor cortex in adult macaque monkeys with long-standing amputations. J. Neurophysiol. 84, 2133-2147.

Rockel, A. J., Hiorns, R. W., Powel, T. P. (1980). The basic uniformity in the structure of the neocortex. Brain 103, 221-244.

Sessle, B. J., and Wiesendanger, M (1982). Structural and functional definition of the motor cortex in the monkey (Macaca fascicularis). J. Physiol. 323, 245-265.

Sherwood, C. C., Holloway, R. L., Erwin, J. M., and Hof, P. R. (2004) Cortical orofacial motor representation in old world monkeys, great apes, and humans. II. Stereological analysis of chemoarchitecture. Brain Behav. Evol. 63, 82-106.

Sherwood, C. C., Holloway, R. L., Gannon, P. J., Semendeferi, K., Erwin, J. M., Zilles, K., et al. (2003). Neuroanatomical basis of facial expression in monkeys, apes, and humans. Ann. N.Y. Acad. Sci. 1000, 99-103.

Sherwood, C. C., Raghanti, M. A., Stimpson, C. D., Spocter, M. A. Uddin, M., Boddy, A. M., et al. (2010). Inhibitory interneurons of the uman prefrontal cortex display conserved evolution of the phenotype and related genes. Proc. Biol. Sci. 277, 1011-1120.

Skoglund, T. S., Pascher, R. and Berthold, C. H. (1996). Heterogeneity in the columnar number of neurons in different neocortical areas in the rat. Neurosci. Lett. 208, 97-100.

Stepniewska, I., Fang, P. C., and Kaas, J. H. (2005). Microstimulation reveals specialized subregions for different complex movements in posterior parietal cortex of prosimian galagos. Proc. Natl. Acad. Sci. U.S.A. 102 , 4878-4883.

Stepniewska, I., Fang, P. Y., and Kaas, J. H. (2009). Organization of the posterior parietal cortex in galagos: 1. Functional zones identified by microstimulation. J. Comp. Neurol. $517,765-782$

Stepniewska, I., Preuss, T. M., and Kaas, J. H. (1993). Architectonics, somatotopic organization, and ipsilateral cortical connections of the primary motor area (M1) of owl monkeys. J. Comp. Neurol. 330, 238-271

Van Essen, D. C., Glasser, M. F., Dierker, D. L., and Harwell, J. (2011). Cortical parcellations of the macaque monkey analyzed on surface-based atlases. Cereb. Cortex 22, 2227-2240.

Waters, R. S., Samulack, D. D., Dykes, R. W., and McKinley, P. A. (1990). Topographic organization of the baboon primary motor cortex: face, hands, forelimb, and shoulder representations. Somatosens. Mot. Res. 7, 485-514.

Wise, S. P., and Tanji, J. (1981). Supplementary and precentral motor cortex: contrast in responsiveness to peripheral input in the hindlimb area of the unanesthetized monkey. J. Comp. Neurol. 195, 433-451.

Wong, P., and Kaas, J. H. (2010). Architectonic subdivisions of neocortex in the Galago (Otolemur gar netti). Anat. Rec. 293, 1033-1069.

Wu, C. W.-H., Bichot, N. P., and Kaas, J. H. (2000). Converging evidence from microstimulation, architecture, and connections for multiple motot areas in the frontal and cingulate cortex of prosimian primates. J. Comp. Neurol. 423, 140-177.

Wu, C. W.-H., and Kaas, J. H. (2003). Somatosensory cortex of prosimian Galagos: physiological recording, cytoarchitecture and corticocortical connections of anterior parietal cortex and cortex of the lateral sulcus. J. Comp. Neurol. 457, 263-292.

Young, N. A., Flaherty, D. K., Airey, D. C., Varlan, P., Aworunse, F., Kaas, J. H., et al. (2012). Use of flow cytometry for high-throughput cell population estimates in brain tissue. Front. Neuroanat. 6:27. doi: 10.3389/fnana.2012.00027

Young, N. A., Vuong, J., Flynn, C., and Teskey, G. C. (2011). Optimal parameters for microstimulation derived forelimb movement thresholds and motor maps in rats and mice. J. Neurosci. Methods 196, 60-69.

Conflict of Interest Statement: The authors declare that the research was conducted in the absence of any commercial or financial relationships that could be construed as a potential conflict of interest.

Received: 30 November 2012; accepted: 08 February 2013; published online: 27 February 2013.

Citation: Young NA, Collins CE and Kaas JH (2013) Cell and neuron densities in the primary motor cortex of primates. Front. Neural Circuits 7:30. doi: 10.3389/ fncir.2013.00030

Copyright (c) 2013 Young, Collins and Kaas. This is an open-access article distributed under the terms of the Creative Commons Attribution License, which permits use, distribution and reproduction in other forums, provided the original authors and source are credited and subject to any copyright notices concerning any third-party graphics etc. 